\title{
Fundamental Studies on Poly(2-oxazoline) Side Chain Isomers Using Tandem Mass Spectrometry and Ion Mobility-Mass Spectrometry
}

\author{
Jean R. N. Haler, ${ }^{1}$ @ Victor R. de la Rosa, ${ }^{2}$ Philippe Massonnet, ${ }^{1}$ Johann Far, ${ }^{1}$ \\ Richard Hoogenboom, ${ }^{2}$ Edwin De Pauw ${ }^{1}$ \\ ${ }^{1}$ Mass Spectrometry Laboratory, MolSys Research unit, Quartier Agora, University of Liège, Allée du Six Aout 11, B-4000, Liège, \\ Belgium \\ ${ }^{2}$ Supramolecular Chemistry Group, Centre of Macromolecular Chemistry (CMaC), Department of Organic and Macromolecular \\ Chemistry, Ghent University, Krijgslaan 281 S4, B-9000, Ghent, Belgium
}

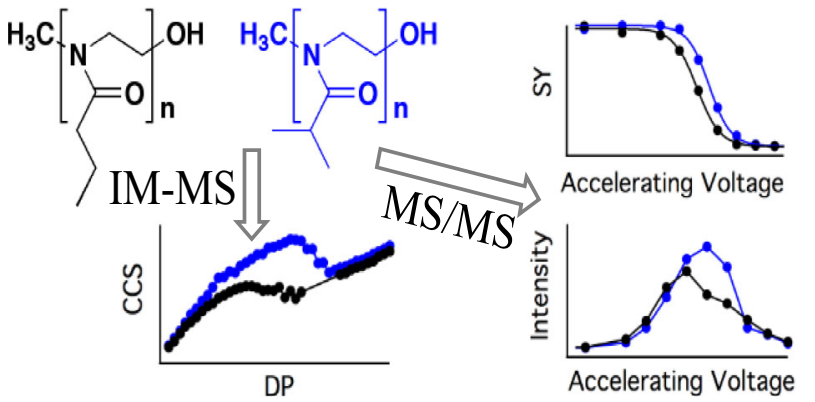

Abstract. When polymer mixtures become in-
creasingly complex, the conventional analysis
techniques become insufficient for complete
characterization. Mass spectrometric techniques
can satisfy this increasing demand for detailed
sample characterization. Even though isobaric
polymers are indistinguishable using simple
mass spectrometry (MS) analyses, more ad-
vanced techniques such as tandem MS (MS/
MS) or ion mobility (IM) can be used. Here, we report proof of concept for characterizing isomeric polymers, namely poly(2-n-propyl-2-oxazoline) (Pn-PrOx) and poly(2-isopropyl-2-oxazoline) (Pi-PrOx), using MS/MS and IM-MS. Pi-PrOx ions lose in intensity at higher accelerating voltages than $\mathrm{P} n$-PrOx ions during collision-induced dissociation (CID) MS/MS experiments. A $\mathrm{Pn} /$ /-PrOx mixture could also be titrated using survival yield calculations of either precursor ions or cation ejection species. IM-MS yielded shape differences in the degree of polymerization (DP) regions showing the structural rearrangements. Combined MS techniques are thus able to identify and deconvolute the molar mass distributions of the two isomers in a mixture. Finally, the MS/MS and IM-MS behaviors are compared for interpretation.

Keywords: Poly(2-oxazoline)s, Synthetic polymer isomers, Tandem mass spectrometry MS/MS, Collisioninduced dissociation, Ion mobility-mass spectrometry

Received: 11 August 2018/Revised: 14 February 2019/Accepted: 25 February 2019/Published Online: 4 April 2019

\section{Introduction}

ize exclusion chromatography (SEC) and nuclear magnetic $\checkmark$ resonance (NMR) have been incorporated since several decades in routine polymer structure characterization [1]. These techniques provide information on the average polymer chain length, the molar mass distribution, and chemical

Electronic supplementary material The online version of this article (https:// doi.org/10.1007/s13361-019-02173-y) contains supplementary material, which is available to authorized users.

Correspondence to: Jean Haler; e-mail: jean.haler@uliege.be structure based on the measurement of hydrodynamic volumes and chemical environments of atoms, mostly protons and carbons, respectively. When these conventional techniques (e.g., SEC, NMR) are unable to resolve the presence of polymer topology or branching contaminations in samples due to signal overlap or due to signal broadenings potentially hiding less intense signals, mass spectrometric techniques (MS) may help with sample characterization.

In such cases (complex mixtures, isobaric compounds), tandem mass spectrometry (MS/MS) experiments bring help for sample characterizations. The most commonly used MS/ MS ion activation technique is collision-induced dissociation (CID). For instance, cyclic and linear polymers could be 
distinguished [2-6] by using MS/MS, different isobaric polymer mixtures could be resolved [7], and different polymer types could be distinguished according to their accelerating voltage at $50 \%$ survival yield (SY) of the precursor ions $\left(V_{50}\right.$; using a quadrupole ion trap instrument) [4].

If the spectra are still too complex to be resolved by MS/MS only, or if the fragments or $V_{50}$ values are not different enough to draw unambiguous conclusions on the presence of different polymer contaminations in a sample, then additional separation techniques can be coupled to the mass spectrometer. Ion mobility-MS (IM-MS) counts among the most recent couplings to be probed for polymer characterizations [8-19]. Even further, MS couplings such as liquid chromatography (LC) LCIM-MS are beginning to be investigated [20].

In the polymer field, structurally similar to natural poly(peptide)s, poly(2-alkyl/aryl-2-oxazoline)s (PAOx) have attracted significant attention since the last five decades and are subject to a renewed interest, especially in the biomedical field [21, 22]. The increasing compositional and architectural complexity of PAOx and other synthetic polymers developed for advanced applications is giving rise to analytical challenges as the conventional techniques fall short of providing a complete description of the polymer structure (e.g., co-monomer sequence, polymer topologies and branching, isomer identification). PAOx have been the subject of a number of in-depth analytical studies involving mass spectrometry, shedding light on fragmentation pathways [23-25], polymerization mechanism [26, 27], and unveiling chain transfer reactions [28, 29] or the mechanisms associated with polymer degradation [30].

Here, we establish the proof of concept for resolving two pure side chain isomers of PAOx [31, 32], namely poly(2-npropyl-2-oxazoline) ( $\mathrm{P} n$-PrOx) and poly(2-iso-propyl-2oxazoline) ( $\mathrm{P} i$-PrOx), using mass spectrometric techniques (MS/MS and IM-MS). First, CID MS/MS experiments are undertaken on the pure samples. A $\mathrm{P} n$-PrOx and $\mathrm{P} i$-PrOx mixture is then analyzed and semi-quantitatively titrated using CID. Then, IM-MS experiments provide a second separation dimension of the two isomeric polymers. Finally, the IM-MS experiments allow understanding and interpreting the observed MS/MS behavior.

\section{Materials and Methods}

\section{Polymers}

Poly(2-n-propyl-2-oxazoline) Pn-PrOx and poly(2-isopropyl2-oxazoline) Pi-PrOx (Figure 1) were synthesized according to (a)

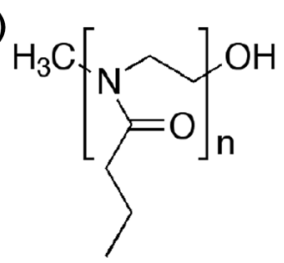

(b)

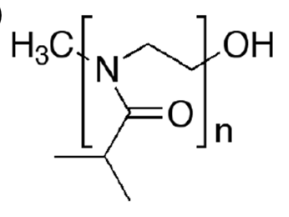

Figure 1. Representation of the chemical structures of (a) PnPrOx and (b) Pi-PrOx the methodology previously reported [33]. The polymerization times were calculated using the reported polymerization rate constant for each monomer to reach a monomer conversion of 99\%. After termination with methanolic tetramethyl ammonium hydroxide, the solvent was removed under reduced pressure resulting in a white solid. The polymers were re-dissolved in water, purified by dialysis in demineralized water (SpectrumLabs dialysis tubing, MWCO 100-500 Da) and freeze-dried. Polymer characterization was performed by ${ }^{1} \mathrm{H}$ NMR spectroscopy, MALDI-ToF mass spectrometry, and size exclusion chromatography (see Supplementary Information, Figure SI1). Five $\mathrm{P} n$-PrOx $\left(\mathrm{DP}_{\text {average }}=20,40,50,2 \times 100\right)$ and four Pi-PrOx $\left(\mathrm{DP}_{\text {average }}=20,30,40,50\right)$ samples were analyzed to cover the mass ranges in this study. The samples yielded overlaps in their mass distributions (i.e., synthesized DP ranges) in order to produce multiple replicate measurements of most of the IM-MS data points.

\section{Ion Mobility-Mass Spectrometry}

$\mathrm{P} n$-PrOx and $\mathrm{P} i$-PrOx samples were dissolved in pure methanol and spiked with $\mathrm{Na}^{+}$cations $(\mathrm{NaCl}$ salt) in order to yield solution concentrations of $10^{-6}-10^{-5} \mathrm{M}$ for MS infusions. The ion mobility-mass spectrometer used for this study was a Traveling Wave (T-Wave) Synapt G2 HDMS (Waters, UK). Detailed experimental conditions can be found in the Supplementary Information.

The analysis of the IM-MS data was performed using Waters' MassLynx 4.1 software. IM arrival time distributions (ATD) were fitted with Gaussian functions using PeakFit v.4.11 to determine accurate drift times. Data processing was performed using Excel 2011 and IgorPro 6.37.

\section{IM-MS Collision Cross-Section Calculation}

The T-Wave IM cell has to be calibrated in order to convert the drift times into CCS values. The calibration protocol from literature was followed [34] (for details on the calibrating substances and the calibration curves, see the Supplementary Information (Figure SI2 and Figure SI3)).

\section{MS/MS (CID) Experiments}

The MS/MS CID experiments were performed using the "trap bias" as accelerating voltage (set from 45 to $200 \mathrm{~V}$ ). These electrodes are located between the end of the trap cell, which forms the ion packets for IM separation, and the entrance of the He cell, which is located in front of the IM cell [35].

\section{Survival Yield and Relative Intensity Calculations}

The SY [2, 4, 7, 36-38] represents the ion intensity of a selected precursor ion which is normalized to all ion signals at a given acceleration voltage (Eq. 1). The SYs can be calculated for CID experiments sampling different accelerating voltages, yielding the SY curves. They can typically be fitted with sigmoid functions (Eq. 2). In this study, only isobaric ions are compared, which allows us to directly compare accelerating 
voltages. From the sigmoid functions, we can calculate the accelerating voltage at $50 \%$ of $\mathrm{SY}$, called $\mathrm{V}_{50}$, in order to compare the results of the two polymers.

$$
\mathrm{SY}=\frac{I_{\text {precursor }}}{I_{\text {precursor }}+\sum I_{\text {fragments }}}
$$

where SY represents the survival yield, $I_{\text {precursor }}$ is the signal intensity of the precursor ion, and $\Sigma I_{\text {fragments }}$ are the signal intensities of all other ions in the spectrum (fragments).

$$
\mathrm{SY}=\text { base }+\frac{\max }{1+\exp \left(\frac{\text { xhal }-x}{\text { rate }}\right)}
$$

where SY is the survival yield plotted as a function of the accelerating voltage, base sets the SY at small voltages, base + max sets the SY at large voltages, xhalf sets the voltage at value (base $+\max ) / 2, x$ represents the voltage, and rate sets the decrease rate of the curve ("slope"). The parameters base and max allow for small experimental SY offsets.

The calculation of the relative intensities of fragment ions when sampling different acceleration voltages is also performed using Eq. 1. In this case, $I_{\text {precursor }}$ is the intensity of any fragment ion and the denominator still represents the intensities of all ions in the spectrum. The intensities of the formed fragment ions do not follow a sigmoid function when plotted as a function of the accelerating voltage. The intensity of the fragments first increases (fragment formation at small voltages) before decreasing (high voltages), rather yielding Gaussian functions. In this study, we do not proceed to fit the fragment ion intensities according to the accelerating voltage.

\section{Thermogravimetric Analysis}

Details are reported in the Supplementary Information.

\section{${ }^{1}$ H Nuclear Magnetic Resonance}

Details are reported in the Supplementary Information.

\section{Size Exclusion Chromatography}

Details are reported in the Supplementary Information.

\section{Results and Discussions}

CID fragments and fragmentation mechanisms of PAOx have been extensively described in literature [23-25, 36, 39]. They ranged from mechanisms such as depolymerizations and side chain losses to McLafferty rearrangements, depending on the side chains and the chain end functions. We then first attempted to discriminate $\mathrm{P} n$-PrOx and $\mathrm{P} i \mathrm{PrOx}$ ions on the basis of the MS/MS spectra (see Figure SI5 and Figure SI6 in the Supplementary Information). They showed, as expected, identical CID spectra due to identical chain end functions and isomeric side chains. Therefore, fragment ion identifications cannot yield much information on the identity of the precursor side chain isomer. Consequently, we analyzed fragment ion intensities as a function of the accelerating voltage, relating the CID experiments to stability titrations of the ions.

\section{CID MS/MS: Survival Yields of Precursor Ions}

The SY calculation of the precursor ions yields discriminatory information on the two side chain isomeric polymers. The SYs were monitored for the $2+$ and $3+$ charge states (sodiated ions) and for several degrees of polymerization ( $\mathrm{DP}=11,15,20,25$, 27, 29; see Figure 2).

The first observation to be noted from Figure 2 and Table 1 ( $V_{50}$ values) is the increase in the accelerating voltage as a function of the DP for a given charge state and polymer (see also Figure SI7 for the evolution of $V_{50}$ values as a function of the DP). This is generally observed for the center-of-mass energy when increasing the number of normal modes of vibration (or degrees of freedom DOF), in this case when the polymer chain length increases $[4,37,40]$. The more the number of normal modes of vibration (NMVs) or DOFs increases, the more internal energy can be stored in the ions. This leads to an increasing accelerating voltage before reaching the bond dissociation energy barriers.

Second, for all monitored precursor ions, $[\mathrm{P} i$-PrOx + $\left.\mathrm{nNa}^{+}\right]^{\mathrm{n}+}$ ions are kinetically more stable (i.e., within the reaction timeframe sampled by the mass spectrometer) than their $\left[\mathrm{P} n-\mathrm{PrOx}+\mathrm{nNa}^{+}\right]^{\mathrm{n}+}$ counterparts having identical charge states and DPs and they degrade at higher accelerating voltages (Figure 2 and Figure SI7). The difference in $V_{50}$ (accelerating voltage at 50\% survival yield; Eq. 2) of the two pure isomers reaches up to $7-8 \mathrm{~V}$ for the sampled DPs and charge states $(8 \mathrm{~V}$ for $\left[\mathrm{PAOx}_{29}+3 \mathrm{Na}^{+}\right]^{3+}$, see Table 1 ).

This observation leads to the possibility of semiquantitatively titrating a mixture of the two isobaric polymers using their SY curves and $V_{50}$ values. As the $\mathrm{P} n$-PrOx and $\mathrm{P} i$ PrOx polymers are only side chain isomers, one should nevertheless not hope for the observation of a voltage range with constant SY in the $\mathrm{SY}_{\text {mixture }}$ as it has been observed for polymers differing in the nature of their monomer backbone [7] or for polymers having radically different $3 \mathrm{D}$ structures (e.g., linear and cyclic) [3]. In such cases, one of the polymers' SY curve reached zero intensity before the second polymer SY curve began decreasing. In the present study, the $\mathrm{P} n / i-\mathrm{PrOx} V_{50}$ difference is not large enough to generate such a voltage range of constant SY (or stepwise degradation) for easy calculation of the percentage of $\mathrm{P} n$-PrOx and $\mathrm{P} i$-PrOx contained in the mixture. Figure 3 represents the titration of a $\sim 50 / 50$ mixture of the two side chain isomers for $\mathrm{DP}=20$ bearing 3 sodium cations $\left[\mathrm{PAOx} \mathrm{DP}_{\mathrm{DP}}=20+3 \mathrm{Na}^{+}\right]^{3+}$. As briefly discussed in literature [7], the deconvolution of the $\mathrm{SY}_{\text {mixture }}$ curve (Eq. 2) is only semiquantitative as small variations may result in large interpretation differences. Nevertheless, we were able to deconvolute the $V_{50}$ value $\left(V_{50}\left(\right.\right.$ Mixture $\left.\left._{\mathrm{DP}=20}\right)=127 \mathrm{~V}\right)$ using a mathematical equation system (Eqs. 3 and 4 ) based on the two pure-sample $V_{50}$ values $\left(V_{50}\left(\mathrm{P} n-\mathrm{PrOx}_{\mathrm{DP}=20}\right)=124 \mathrm{~V}\right.$ and $V_{50}(\mathrm{P} i-$ 


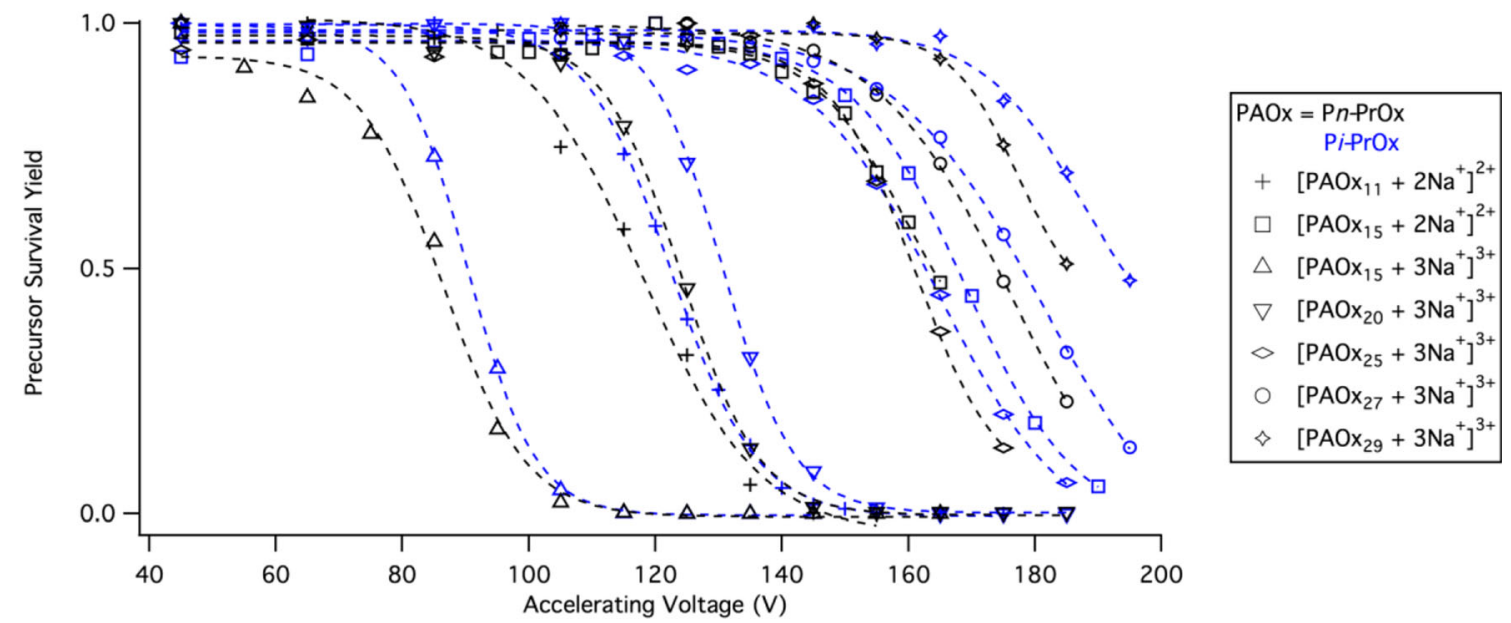

Figure 2. Survival yield (SY) curves (obtained from Eq. 1) of Pn-PrOx (black markers with black dotted lines) and Pi-PrOx (blue markers with blue dotted lines) sodiated precursor ions plotted as a function of the accelerating voltage. The SY curves are recorded for different charge states (2+ and 3+ precursor ions) and different degrees of polymerization (DP = 11, 15, 20, 25, 27, 29). The black and blue lines represent the sigmoid fits of the SY curves (Eq. 2)

$\left.\operatorname{PrOx}_{\mathrm{DP}=20}\right)=131 \mathrm{~V}$, see Table 1 ). The $\mathrm{P} n / i-\mathrm{PrOx} \mathrm{x}_{\mathrm{DP}=20}$ mixture was hence titrated to a mixture containing $60 \%$ of $\mathrm{P} n$ $\mathrm{PrOx}_{\mathrm{DP}=20}$ and $40 \%$ of $\mathrm{P} i$-PrOx ${ }_{\mathrm{DP}}=20$. Given that the dispersities of the two mixed samples $\left(\mathrm{DP}_{\text {average }}=20\right)$ are not perfectly matching, the titrated isomer proportions are in good agreement with our $\sim 50 / 50$ initial mixture.

$$
\left\{\begin{array}{c}
V_{50 \text { Mixture }}=n \cdot V_{50 \mathrm{P} n-\mathrm{PrOx}}+i \cdot V_{50 \mathrm{P} i-\mathrm{PrOx}} \\
1=n+i
\end{array}\right.
$$

$$
\left\{\begin{array}{c}
n=\frac{V_{50 \text { Mixture }}-V_{50} \mathrm{P} i-\mathrm{PrOx}}{V_{50 \mathrm{P} n-\mathrm{PrOx}}-V_{50 \mathrm{P} i-\mathrm{PrOx}}} \\
i=1-n
\end{array}\right.
$$

where $n$ and $i$ represent the proportions of respectively $\mathrm{P} n$ $\mathrm{PrOx}$ and $\mathrm{P} i$-PrOx contained in the mixture.

Comparatively, deconvoluting a $\mathrm{P} n / i$-PrOx mixture can also be performed using ${ }^{1} \mathrm{H}$ NMR spectroscopy (Figure SI8 and Figure SI9). However, ${ }^{1} \mathrm{H}$ NMR spectroscopy only yields average-DP information, without the possibility of deconvoluting the dispersities of the constituting pure $\mathrm{P} n$ - $\mathrm{PrOx}$

Table 1. $V_{50}$ values of $\mathrm{P} n$-PrOx and $\mathrm{P} i$-PrOx extracted from the survival yields (Eq. 1) plotted in Figure 2 using Eq. 2. The SYs were recorded for the 2+ and 3+ sodiated precursor ions at different degrees of polymerization $(\mathrm{DP}=11,15,20$, $25,27,29)$

\begin{tabular}{lll}
\hline Precursor ion DP and charge state & $V_{50}(\mathrm{~V}) \mathrm{P} n$-PrOx & $V_{50}(\mathrm{~V}) \mathrm{Pi}$-PrOx \\
\hline$\left[\mathrm{PAOx}_{11}+2 \mathrm{Na}^{+}\right]^{2+}$ & 117 & 122 \\
{$\left[\mathrm{PAOx}_{15}+2 \mathrm{Na}^{+}\right]^{2+}$} & 164 & 168 \\
{$\left[\mathrm{PAOx}_{15}+3 \mathrm{Na}^{+}\right]^{3+}$} & 86 & 90 \\
{$\left[\mathrm{PAOx}_{20}+3 \mathrm{Na}^{+}\right]^{3+}$} & 124 & 131 \\
{$\left[\mathrm{PAOx}_{25}+3 \mathrm{Na}^{+}\right]^{3+}$} & 161 & 163 \\
{$\left[\mathrm{PAOx}_{27}+3 \mathrm{Na}^{+}\right]^{3+}$} & 174 & 178 \\
{$\left[\mathrm{PAOx}_{29}+3 \mathrm{Na}^{+}\right]^{3+}$} & 186 & 194 \\
\hline
\end{tabular}

and $\mathrm{P} i$-PrOx samples, i.e., deconvoluting each DP in the sample. Furthermore, NMR only allows the deconvolution of complex mixtures (tertiary mixtures, additives...) as long as no chemical shift overlaps take place. A brief discussion and comparison between CID ion heating and macroscopic TGA degradation analyses (Figure SI4) can be found in the Supplementary Information. In short, the pure-sample polymers degrade at different temperatures $\left(\Delta T \sim 30^{\circ} \mathrm{C}\right)$ but the mixture does not exhibit a stepwise degradation, meaning that TGA does not allow for a semi-quantitative deconvolution of a $\mathrm{P} n / i$ PrOx mixture. Neither does SEC: the presence of both polymers in an isomer mixture cannot be elucidated, since the trace of the isomer mixture resembles that of a well-defined pure polymer (see Figure SI10).

\section{CID MS/MS: Cation Ejection Species}

Using CID tandem MS, the differences between the two side chain isomeric polymers go further than the $V_{50}$ quantification using the precursor ions. The relative intensities of specific ions can also help resolving a mixture of the two polymers. Such differences can be seen in the cation ejection species. The cation ejection species are constituted of the intact precursor ions not yet having undergone any fragmentation, but having ejected one or several sodium cations (sum of all the species; see Figure 4) [41].

Figure 4 monitors the relative intensities of the precursor ions (SY curves in Figure 2), of the cation ejection species and of all other ions in the MS/MS spectrum (fragment ions) for different charge states and different DPs of $\mathrm{P} n-\mathrm{PrOx}$ and $\mathrm{P} i$ PrOx. The relative intensities are calculated using Eq. 1 (see Materials and Methods). Figure 4a-c show that at smaller DPs, the $\mathrm{P} i$-PrOx ions undergo more favorably cation ejections than $\mathrm{P} n$-PrOx ions. At higher DP values (Figure $4 \mathrm{~d}-\mathrm{f}$ ), this trend is surprisingly reversed, meaning that $\mathrm{P} n$-PrOx ions eject more intensely sodium cations than $\mathrm{P} i$-PrOx ions. 


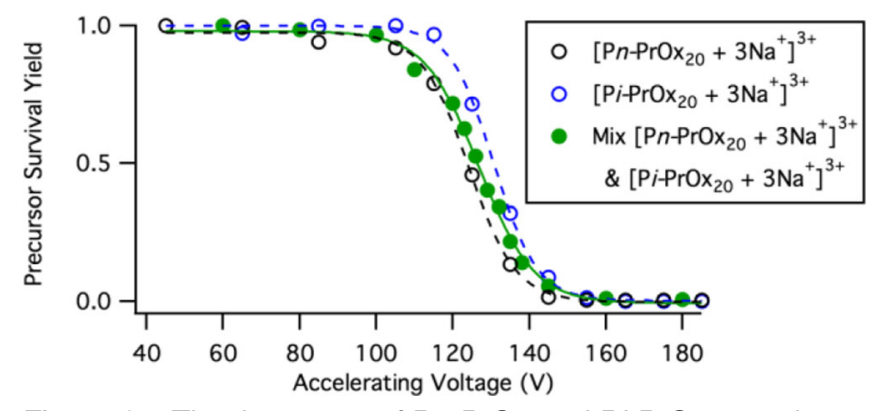

Figure 3. Titration curve of $\mathrm{Pn}-\mathrm{PrOx}$ and $\mathrm{Pi}-\mathrm{PrOx}$ complexes using the example of the $3+$ charge state at DP $=20\left(\left[\mathrm{PAOx}_{20}+\right.\right.$ $\left.\left.3 \mathrm{Na}^{+}\right]^{3+} ; m / z=787.6\right)$. The black markers represent the SY of pure $\mathrm{Pn}$-PrOx precursor ions and the blue markers represent the SY of pure Pi-PrOx ions. The green plain markers represent the SY of the mixture $(\sim 50 / 50)$ of the two pure isomeric polymers. The color-coded lines are the sigmoid fits of the SY curves used for $V_{50}$ calculation (Eq. 2)

In a $\mathrm{P} n / i$-PrOx mixture, the two polymer contributions can hence also be titrated using the cation ejection species. This can be very helpful if the precursor ion $\mathrm{m} / \mathrm{z}$ isolation (e.g., using a quadrupole mass filter) is not efficient enough to yield clean precursor ion selections without any other signal overlaps. This might be the case in spectra where too many ion peaks are present in small $\mathrm{m} / \mathrm{z}$ regions of interest. Due to the decrease in their charge state (loss of cations) leading to higher $\mathrm{m} / \mathrm{z}$ values than the precursor ions, the CID-formed cation ejection species will appear in non-convoluted regions of the spectra where fewer interfering ions are present. This simplifies (or enables) data treatment. Figure SI1 1 illustrates the relative intensities of the cation ejection species from pure $\mathrm{P} n$-PrOx and $\mathrm{P} i$-PrOx samples as well as from the $\sim 50 / 50$ mixture. Using the maximum of the relative intensities $\left(I_{\max }(\mathrm{P} n-\mathrm{PrOx})=0.62 ; I_{\max }\right.$ $(\mathrm{P} i$-PrOx $)=0.82 ; I_{\max }($ mixture $\left.)=0.70\right)$, the mixture can be titrated as containing $60 \%$ of $\mathrm{P} n-\mathrm{PrOx}$ and $40 \%$ of $\mathrm{P} i$-PrOx. This is in excellent agreement with the previous SY deconvolution of the precursor ions (60\% $\mathrm{P} n-\mathrm{PrOx}$ and $40 \%$ $\mathrm{P} i$-PrOx).

\section{IM-MS: CCS Evolutions}

Aside from the different MS/MS deconvolutions of the two PAOx polymers, shape information acquired through IM coupled to the mass spectrometric identification of the ions (IM-MS) also yields a discriminatory descriptor of the two side chain isomeric polymers. The CCS evolutions plotted as a function of the DP of $\mathrm{P} n-\mathrm{PrOx}$ and $\mathrm{P} i$-PrOx for different charge states $(1+$ to $4+)$ are represented in Figure 5.

In general, the CCS evolutions of polymer ions [10, 42-44] are dictated by enthalpic and entropic factors. These enthalpic and entropic factors originate from, e.g., the Coulomb repulsions (cation charges), the charge solvation capacities of the chemical functions, steric hindrance of the polymer chain, and the entropy drive to yield the most entangled (compact) polymer chains.
As already reported for other polymers [15, 42-45], the CCS increases monotonically for a given charge state when the DP increases. The $1+$ and $2+$ ions follow the same CCS trend, which represents the most compact shape of the polymer-sodium complex, as exhibited in Figure 5. When increasing the charge state from the $2+$ to the $3+$ or $4+$ charge states at small DP values, the CCS increases due to the increase in Coulomb repulsions which distort the shape of the polymer ions. When the DP increases for the $3+$ or $4+$ charge states, the CCS evolutions exhibit tipping points [44] at higher DP values. At these tipping points, the CCS does not increase at the same rate anymore and may even decrease, as exhibited by the $4+$ charge state. Here, the polymer is able to stabilize (solvate) the cations more efficiently by adopting a different shape, i.e., by undergoing a structural rearrangement. After the structural rearrangements, the CCS evolutions merge with the most compact, entropically favored CCS evolution.

In the case of $\mathrm{P} n$-PrOx and $\mathrm{P} i$-PrOx, the CCS evolutions before and after the structural rearrangements are very similar. The most compact CCS evolution [44] is also very similar for the two polymers. The differences in solvation capacities due to the isomeric side chains only clearly appear during the structural rearrangements $(3+$ and $4+$ charge states; Figure 5 or Figure SI12). $\left[\mathrm{P} n-\mathrm{PrOx}+3 \mathrm{Na}^{+}\right]^{3+}$ ions rearrange from DP 21 to 27 whereas $\left[\mathrm{Pi} \text {-PrOx }+3 \mathrm{Na}^{+}\right]^{3+}$ ions rearrange from DP 28 to DP 32. The structural rearrangement of $\mathrm{P} i$-PrOx occurred at higher DP values and even higher CCS values than the structural rearrangement of $\mathrm{P} n$-PrOx for the $4+$ charge state compared to the lower charge state (see Figure 5). During these DP ranges, the balance between Coulomb repulsions, favoring larger ion shapes, and charge solvation and entropic factors, favoring more compact shapes, is playing a crucial role. $\mathrm{P} n$ PrOx rearranges at smaller DP values than $\mathrm{P} i$-PrOx ions, meaning that $\mathrm{P} i$-PrOx does not solvate the cations as easily as $\mathrm{P} n$-PrOx in the gas phase. $\mathrm{P} i$-PrOx thus exhibits a reduced cation solvation efficiency compared to $\mathrm{P} n-\mathrm{PrOx}$. This result suggests that the isopropyl groups hinder the accessibility of the amide function solvating the cation charges.

Ion mobility can thus be used for $\mathrm{P} n / i$-PrOx mixture characterizations if the DP values of structural rearrangements can be sampled (for $3+, 4+$ or higher charge states). Figure 6 illustrates the pure $\mathrm{P} n$-PrOx and $\mathrm{P} i$-PrOx ATDs and the convoluted ATD [46] of a Pn/i-PrOx mixture, which can be deconvoluted into the two pure-polymer ATD peaks (different $\mathrm{P} n / i$-PrOx sample mixture than for MS/MS experiments, leading to a mixture closer to 50/50 in this case when deconvoluting the mixture using Gaussian area values).

\section{Crossing MS/MS and IM-MS Information}

$\mathrm{P} i$-PrOx ions decreasing in intensity at higher $V_{50}$ values (Figure 2) compared to $\mathrm{P} n$ - $\mathrm{PrOx}$ ions could at a first glance seem contradictory. Given the increased stability of an isopropyl carbocation fragment ion compared to the $n$-propyl counterpart, one could expect that $\mathrm{P} i$-PrOx should degrade easier at lower accelerating voltages. However, literature fragmentation 


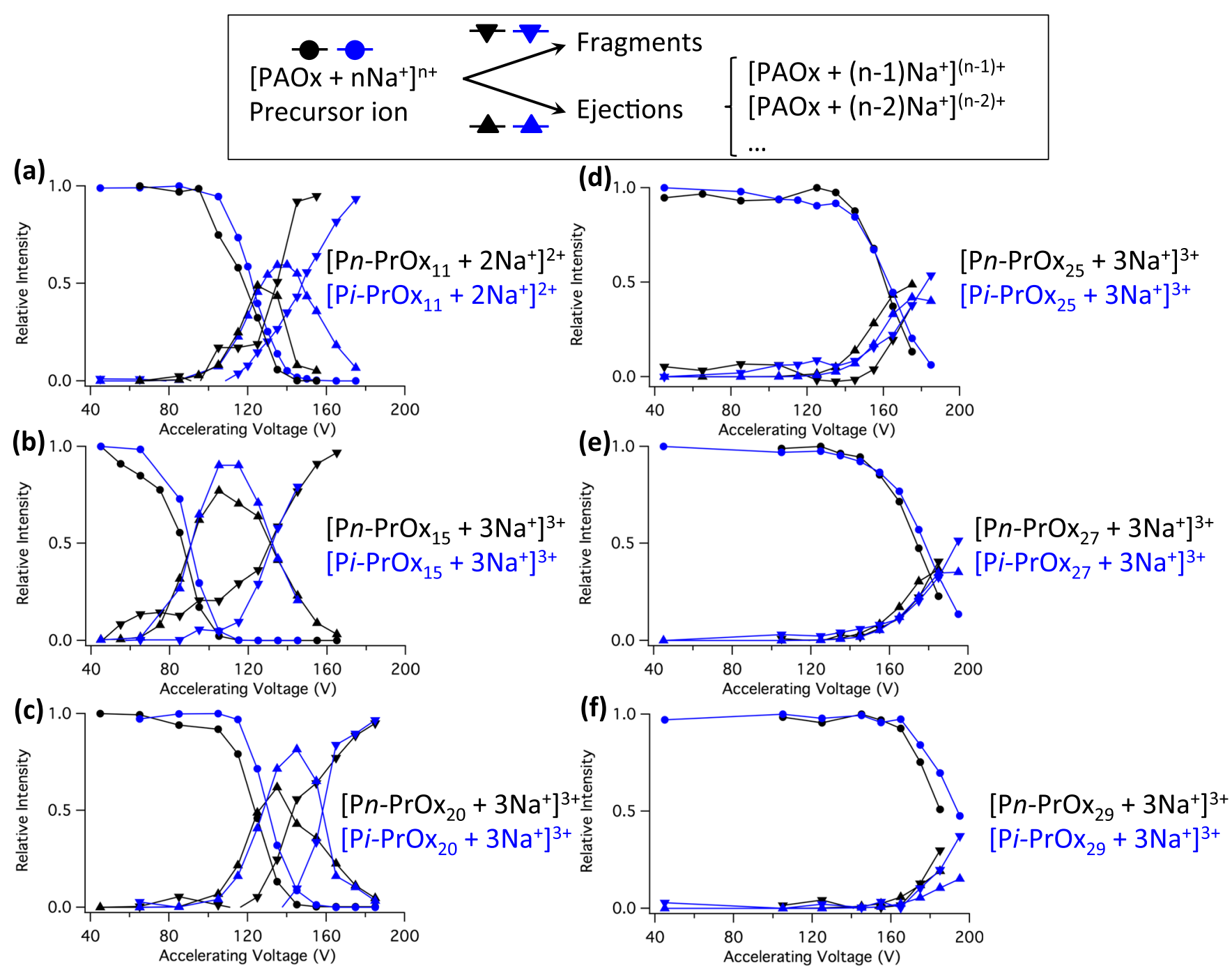

Figure 4. Relative signal intensities (Eq. 1) plotted as a function of the accelerating voltage. In each plot, the black markers and lines represent $\mathrm{Pn}$-PrOx ions and the blue markers and lines represent $\mathrm{Pi}$-PrOx ions. The precursor ions were sampled as $2+$ and $3+$ charge states for different DP values. The circle markers represent the SY of the precursor ions, the upwards-facing triangles represent the intact polymers having lost sodium cations (ejection) and the downwards-facing triangles represent all other ions (fragmentation) in the spectra. Panels (a)-(f) show the SY plots for different DPs of Pn-PrOx and Pi-PrOx: the $\left[\mathrm{Pn} / i-\mathrm{PrOx}_{11}+2 \mathrm{Na}^{+}\right]^{2+}$,

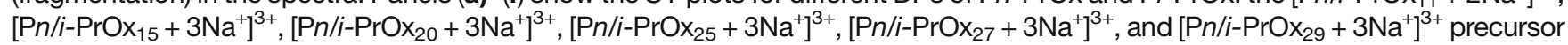
ions, respectively

mechanisms report that the fragments originating from side chain losses also contain the oxygen atom of the amide bonds [23]. This solvating oxygen atom cancels out the carbocation stability hypothesis for the two side chain isomers. Therefore, the main factor responsible for the increased precursor ion stability of Pi-PrOx should be the inductive effect [47] of the isopropyl side chains, or simply the decreased flexibility which could shield the polymer backbone and hence increase its stability, which is subjected to the main fragmentation mechanisms according to literature [23-25, 36, 39].

When analyzing in detail the signal intensities of the cation ejection species (cation losses) as a function of the DP for the two polymers (Figure 4), it can be noted that $\mathrm{P} i$-PrOx ejects more favorably (higher ion intensity) sodium cations until $\left[\mathrm{PAOx}_{20}+3 \mathrm{Na}^{+}\right]^{3+}$ (Figure $4 \mathrm{a}-\mathrm{c}$ ), even if its precursor ions degrade at higher accelerating voltages. This means that once enough energy is conferred to the ions for $\mathrm{P} i$-PrOx to start degrading, its sodium cations are observed to be less efficiently stabilized by the polymer chain. Around the $\left[\mathrm{PAOx}_{25}+\right.$ $\left.3 \mathrm{Na}^{+}\right]^{3+}$ complexes, the intensities of the cation ejection species are reversed and $\mathrm{P} n$-PrOx ejects more easily sodium cations (Figure $4 \mathrm{~d}-\mathrm{f}$ ). This behavior can be correlated to the IMMS behavior of the two polymers. As long as the $\mathrm{P} n / i-\mathrm{PrOx}$ CCS evolutions are almost confounded ( $3+$ charge state, DP $<$ 22; see Figure 5 and Figure SI12), Pi-PrOx more easily ejects its sodium cations. When the shapes of the $[\mathrm{P} n / i-\mathrm{PrOx}+$ $\left.3 \mathrm{Na}^{+}\right]^{3+}$ ions then begin to differ (DP 22-32; see Figure 5 and Figure SI12), the P $n$-PrOx polymer ejects more easily the cations. If higher accelerating voltages were accessible in the mass spectrometer used for this study, we should find a second 


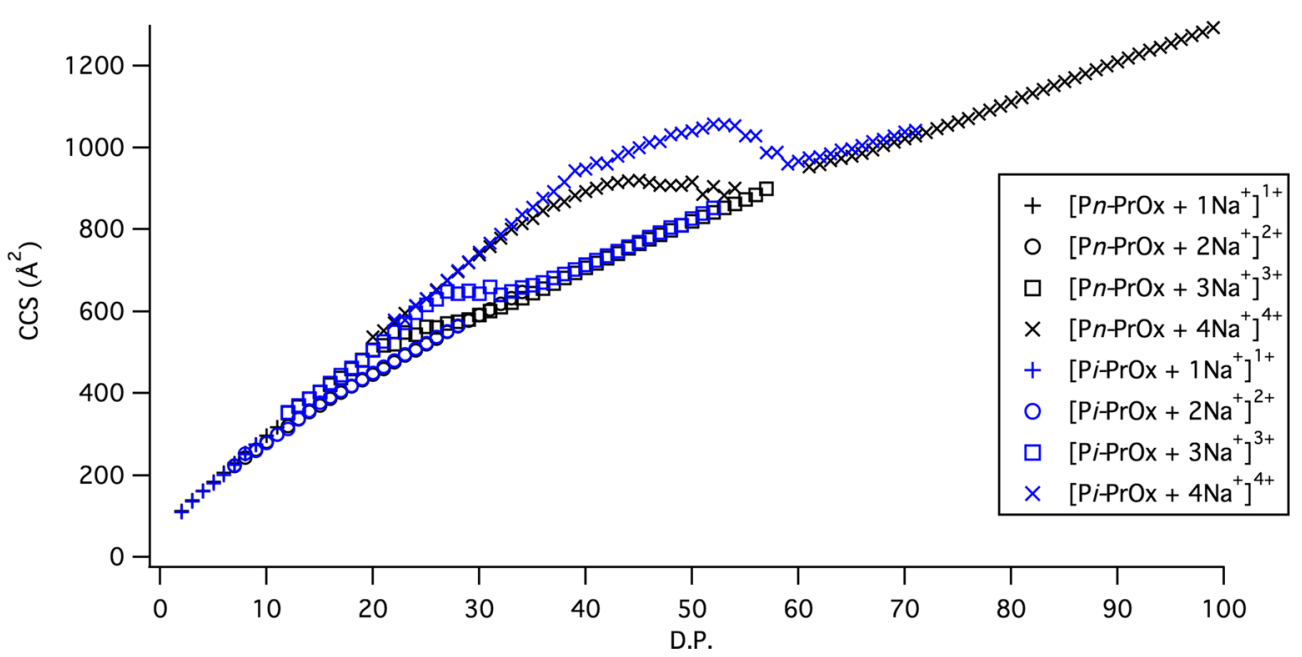

Figure 5. Collision cross-section (CCS) plotted as a function of the degree of polymerization (DP) of pure Pn-PrOx (black markers) and $\mathrm{Pi}$-PrOx (blue markers) samples. The interpreted charge states range from $\left[\mathrm{PAOx}+1 \mathrm{Na}^{+}\right]^{1+}$ to $\left[\mathrm{PAOx}+4 \mathrm{Na}^{+}\right]^{4+}$

inversion in the cation ejection intensities once the two polymers follow again similar CCS evolutions (DP > 32). This behavior has its origins in the ejection dynamics which depend on the 3D structure. As already discussed in literature, when the 3D structure changes, the ejection dynamics change [48] (DP 22-32), leading to the correlation between the observed MS/ MS and IM-MS behaviors.

\section{Conclusions}

In our proof of concept, MS/MS and IM-MS analyses allowed for signal deconvolutions of each DP of the side chain isomeric polymers, namely poly(2-n-propyl-2-oxazoline) $\mathrm{P} n$-PrOx and poly(2-isopropyl-2-oxazoline) $\mathrm{P} i$-PrOx, in a $\mathrm{P} n / i$-PrOx

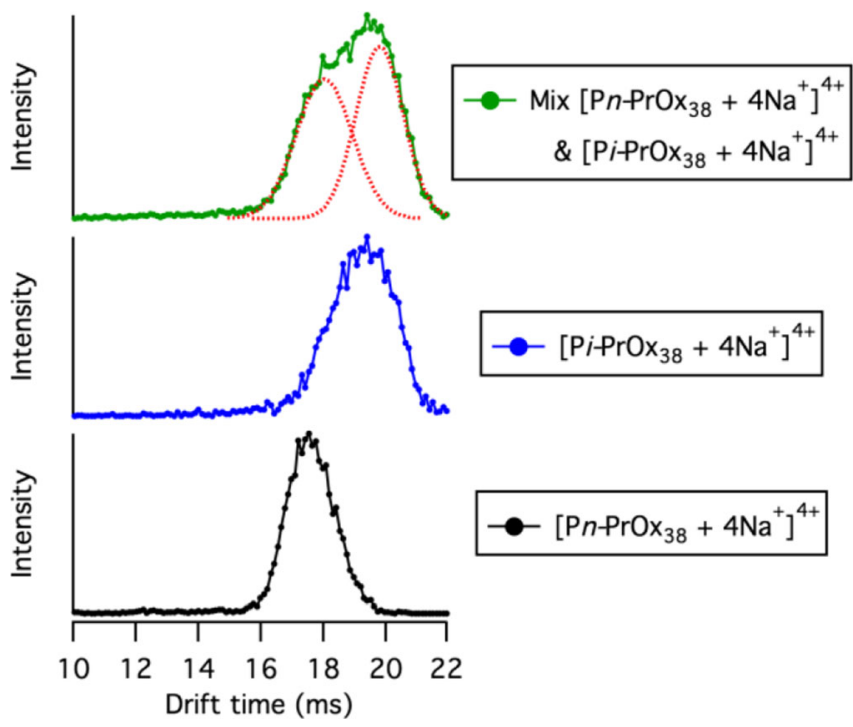

Figure 6. Arrival time distributions (ATDs) of pure $\left[\mathrm{Pn}\right.$ - $\mathrm{PrOx}_{38}+$ $\left.4 \mathrm{Na}^{+}\right]^{4+}$ (black trace) and pure $\left[\mathrm{Pi}-\mathrm{PrOx}_{38}+4 \mathrm{Na}^{+}\right]^{4+}$ (blue trace) polymers. The green trace represents the ATD of a mixture of $\left[\mathrm{Pn}-\mathrm{PrOx}_{38}+4 \mathrm{Na}^{+}\right]^{4+}$ and $\left[\mathrm{Pi}-\mathrm{PrOx}_{38}+4 \mathrm{Na}^{+}\right]^{4+}$ and the dotted red curves represent its Gaussian fit deconvolution mixture. This enables the reconstruction of the individual pure-sample dispersities.

Regarding tandem MS experiments (MS/MS), given that the CID fragment ions of both polymers were identical, we focused on survival yield calculations and on the interpretation of relative intensities of specific ions. For all the investigated charge states and DP values, Pi-PrOx ions were found to degrade at higher $V_{50}$ values than $\mathrm{P} n-\mathrm{PrOx}$ ions. This was attributed to the increased inductive effect of the isopropyl side chain compared to the $n$-propyl side chain. Isopropyl side chains stabilize or shield (through a lower flexibility) the polymer backbone, inducing an increase in the precursor ion stabilities. This was used to semi-quantitatively titrate a $\mathrm{P} n / i-\mathrm{PrOx}$ mixture by MS/MS. The $V_{50}$ of the SYs of the mixture precursor ion contributions was titrated owing to the $V_{50}$ values of the SYs of the pure samples.

When analyzing the MS/MS cation ejection species, i.e., intact polymer ions having ejected one or several (sodium) cations, $\mathrm{P} i$-PrOx was found to eject or lose more easily sodium cations at small DP values than $\mathrm{P} n$-PrOx. This trend was reversed at higher DP values. The cation ejection species were then also used to semi-quantitatively titrate (or deconvolute) a $\mathrm{P} n / i$-PrOx mixture. If the spectra of the precursor ions are too congested to interpret the precursor ion signal intensities, cation ejection species can allow data interpretation because they will be found in different regions of the mass spectra where no other ions interfere.

The IM-MS CCS evolutions of both polymers were very similar except in the DP regions of their structural rearrangements. $\mathrm{P} i$-PrOx exhibited structural rearrangements taking place at higher DP values than $\mathrm{P} n$-PrOx, indicating that the charge-solvating amide functions of $\mathrm{P} i$-PrOx are less accessible than the amide functions of $\mathrm{P} n$-PrOx. $i$-Pr shields the amide function due to a decreased flexibility. When deconvoluting a $\mathrm{P} n / i$-PrOx mixture using IM-MS, the DP values and charge states exhibiting the structural rearrangements need thus to be sampled. On the one hand, IM-MS experiments are less timeconsuming than MS/MS experiments but IM-MS 
deconvolutions of a $\mathrm{P} n / i-\mathrm{PrOx}$ mixture are limited to the DP values of the structural rearrangements. On the other hand, the more time-consuming MS/MS experiments allow deconvoluting an isomer mixture for each DP of $\mathrm{P} n / i-\mathrm{PrOx}$.

Finally, the IM-MS behaviors were correlated to the MS/ MS intensities of the cation ejection species. At similar CCS values, $\mathrm{P} i$-PrOx ejected more cations than $\mathrm{P} n$-PrOx whereas the intensity of the cation ejection species was reversed when the two polymers differed in CCS (or shape).

\section{Acknowledgements}

The authors acknowledge the financial support of the F.R.S.FNRS (FRIA). R. Hoogenboom acknowledges financial support from FWO and Ghent University.

\section{Compliance with Ethical Standards}

Conflict of Interest The authors declare that there are no competing interests.

\section{References}

1. Young, R.J., Lovell, P.A.: Introduction to Polymers. CRC PRESS (2011)

2. Jeanne Dit Fouque, D., Maroto, A., Memboeuf, A.: Purification and quantification of an isomeric compound in a mixture by collisional excitation in multistage mass spectrometry experiments. Anal. Chem. 88, 10821-10825 (2016)

3. Josse, T., De Winter, J., Dubois, P., Coulembier, O., Gerbaux, P., Memboeuf, A.: A tandem mass spectrometry-based method to assess the architectural purity of synthetic polymers: a case of a cyclic polylactide obtained by click chemistry. Polym. Chem. 6, 64-69 (2015)

4. Nasioudis, A., Memboeuf, A., Heeren, R.M.A., Smith, D.F., Vékey, K., Drahos, L., Van Den Brink, O.F.: Discrimination of polymers by using their characteristic collision energy in tandem mass spectrometry. Anal. Chem. 82, 9350-9356 (2010)

5. Li, X., Chan, Y.T., Newkome, G.R., Wesdemiotis, C.: Gradient tandem mass spectrometry interfaced with ion mobility separation for the characterization of supramolecular architectures. Anal. Chem. 83, 1284-1290 (2011)

6. Yol, A.M., Dabney, D.E., Wang, S.F., Laurent, B.A., Foster, M.D., Quirk, R.P., Grayson, S.M., Wesdemiotis, C.: Differentiation of linear and cyclic polymer architectures by MALDI tandem mass spectrometry (MALDI-MS2). J. Am. Soc. Mass Spectrom. 24, 74-82 (2013)

7. Memboeuf, A., Jullien, L., Lartia, R., Brasme, B., Gimbert, Y.: Tandem mass spectrometric analysis of a mixture of isobars using the survival yield technique. J. Am. Soc. Mass Spectrom. 22, 1744-1752 (2011)

8. Gies, A.P., Kliman, M., McLean, J.A., Hercules, D.M.: Characterization of branching in aramid polymers studied by MALDI-ion mobility/mass spectrometry. Macromolecules. 41, 8299-8301 (2008)

9. Bagal, D., Zhang, H., Schnier, P.D.: Gas-phase proton-transfer chemistry coupled with TOF mass spectrometry and ion mobility-MS for the facile analysis of poly(ethylene glycols) and PEGylated polypeptide conjugates. Anal. Chem. 80, 2408-2418 (2008)

10. Trimpin, S., Clemmer, D.E.: Ion mobility spectrometry/mass spectrometry snapshots for assessing the molecular compositions of complex polymeric systems. Anal. Chem. 80, 9073-9083 (2008)

11. Hoskins, J.N., Trimpin, S., Grayson, S.M.: Architectural differentiation of linear and cyclic polymeric isomers by ion mobility spectrometry-mass spectrometry. Macromolecules. 44, 6915-6918 (2011)

12. Morsa, D., Defize, T., Dehareng, D., Jérôme, C., De Pauw, E.: Polymer topology revealed by ion mobility coupled with mass spectrometry. Anal. Chem. 86, 9693-9700 (2014)
13. Foley, C.D., Zhang, B., Alb, A.M., Trimpin, S., Grayson, S.M.: Use of ion mobility spectrometry-mass spectrometry to elucidate architectural dispersity within star polymers. ACS Macro Lett. 4, 778-782 (2015)

14. Haler, J.R.N., Far, J., Aqil, A., Claereboudt, J., Tomczyk, N., Giles, K., Jérôme, C., De Pauw, E.: Multiple gas-phase conformations of a synthetic linear poly(acrylamide) polymer observed using ion mobility-mass spectrometry. J. Am. Soc. Mass Spectrom. 28, 2492-2499 (2017)

15. Haler, J.R.N., Massonnet, P., Chirot, F., Kune, C., Comby-Zerbino, C., Jordens, J., Honing, M., Mengerink, Y., Far, J., Dugourd, P., De Pauw, E.: Comparison of different ion mobility setups using poly (ethylene oxide) PEO polymers: drift tube, TIMS, and T-Wave. J. Am. Soc. Mass Spectrom. 29, 114-120 (2018)

16. Farenc, M., Witt, M., Craven, K., Barrère-Mangote, C., Afonso, C., Giusti, P.: Characterization of polyolefin pyrolysis species produced under ambient conditions by Fourier transform ion cyclotron resonance mass spectrometry and ion mobility-mass spectrometry. J. Am. Soc. Mass Spectrom. 28, 507-514 (2017)

17. Barrère, C., Selmi, W., Hubert-Roux, M., Coupin, T., Assumani, B., Afonso, C., Giusti, P.: Rapid analysis of polyester and polyethylene blends by ion mobility-mass spectrometry. Polym. Chem. 5, 3576-3582 (2014)

18. Liu, X., Cool, L.R., Lin, K., Kasko, A.M., Wesdemiotis, C.: Tandem mass spectrometry and ion mobility mass spectrometry for the analysis of molecular sequence and architecture of hyperbranched glycopolymers. Analyst. 140, 1182-1191 (2015)

19. Hilton, G.R., Jackson, A.T., Thalassinos, K., Scrivens, J.H.: Structural analysis of synthetic polymer mixtures using ion mobility and tandem mass spectrometry. Anal. Chem. 80, 9720-9725 (2008)

20. Shi, C., Gerișlioğlu, S., Wesdemiotis, C.: Ultrahigh performance liquid chromatography interfaced with mass spectrometry and orthogonal ion mobility separation for the microstructure characterization of amphiphilic block copolymers. Chromatographia. 79, 961-969 (2016)

21. Hoogenboom, R.: 50 years of poly(2-oxazoline)s. Eur. Polym. J. 88, 448450 (2017)

22. Viegas, T.X., Fang, Z., Yoon, K., Weimer, R., Dizman, B.: 6 Poly(oxazolines) A2 - Parambath, Anilkumar. In: Engineering of Biomaterials for Drug Delivery Systems, pp. 173-198. Woodhead Publishing (2018)

23. Altuntaş, E., Kempe, K., Crecelius, A., Hoogenboom, R., Schubert, U.S.: ESI-MS \& MS/MS analysis of poly(2-oxazoline)s with different side groups. Macromol. Chem. Phys. 211, 2312-2322 (2010)

24. Altuntaş, E., Weber, C., Kempe, K., Schubert, U.S.: Comparison of ESI, APCI and MALDI for the (tandem) mass analysis of poly(2-ethyl-2oxazoline)s with various end-groups. Eur. Polym. J. 49, 2172-2185 (2013)

25. Baumgaertel, A., Weber, C., Knop, K., Crecelius, A., Schubert, U.S.: Characterization of different poly(2-ethyl-2-oxazoline)s via matrixassisted laser desorption/ionization time-of-flight tandem mass spectrometry. Rapid Commun. Mass Spectrom. 23, 756-762 (2009)

26. Baumgaertel, A., Weber, C., Fritz, N., Festag, G., Altuntaș, E., Kempe, K., Hoogenboom, R., Schubert, U.S.: Characterization of poly(2oxazoline) homo- and copolymers by liquid chromatography at critical conditions. J. Chromatogr. A. 1218, 8370-8378 (2011)

27. Chojnacka, A., Kempe, K., van de Ven, H.C., Englert, C., Hoogenboom, R., Schubert, U.S., Janssen, H.-G., Schoenmakers, P.: Molar mass, chemical-composition, and functionality-type distributions of poly(2oxazoline)s revealed by a variety of separation techniques. J. Chromatogr. A. 1265, 123-132 (2012)

28. Biri, B., Nagy, L., Kuki, Á., Töke Enikö, R., Deák, G., Zsuga, M., Kéki, S.: Collision-induced dissociation study of poly(2-ethyl-2-oxazoline) using survival yields and breakdown curves. J. Mass Spectrom. 48, 1623 (2012)

29. Sahn, M., Bandelli, D., Dirauf, M., Weber, C., Schubert Ulrich, S.: Bifunctional initiators as tools to track chain transfer during the CROP of 2-Oxazolines. Macromol. Rapid Commun. 38, 1700396 (2017)

30. Atılkan, N., Nur, Y., Hacaloglu, J., Schlaad, H.: Direct insertion mass spectrometric analysis of thermal degradation of poly(2-alkyl-2oxazoline). Macromol. Chem. Phys. 213, 945-951 (2012)

31. Verbraeken, B., Lava, K., Hoogenboom, R.: Poly(2-oxazoline)s. In: Encyclopedia of Polymer Science and Technology, pp. 1-51. John Wiley \& Sons, Inc., Hoboken, NJ, USA (2014)

32. Hoogenboom, R.: Poly(2-oxazoline)s: a polymer class with numerous potential applications. Angew. Chemie - Int. Ed. 48, 7978-7994 (2009) 
33. de la Rosa, V.R., Tempelaar, S., Dubois, P., Hoogenboom, R., Mespouille, L.: Poly(2-ethyl-2-oxazoline)-block-polycarbonate block copolymers: from improved end-group control in poly(2-oxazoline)s to chain extension with aliphatic polycarbonate through a fully metal-free ring-opening polymerisation process. Polym. Chem. 7, 1559-1568 (2016)

34. Ruotolo, B.T., Benesch, J.L.P., Sandercock, A.M., Hyung, S.J., Robinson, C.V.: Ion mobility-mass spectrometry analysis of large protein complexes. Nat. Protoc. 3, 1139-1152 (2008)

35. Giles, K., Williams, J.P., Campuzano, I.: Enhancements in travelling wave ion mobility resolution. Rapid Commun. Mass Spectrom. 25, 1559-1566 (2011)

36. Altuntas, E., Weber, C., Schubert, U.S.: Detailed characterization of poly(2-ethyl-2oxazoline)s by energy variable collision-induced dissociation study. Rapid Commun. Mass Spectrom. 27, 1095-1100 (2013)

37. Kuki, Á., Nagy, L., Shemirani, G., Memboeuf, A., Drahos, L., Vékey, K., Zsuga, M., Kéki, S.: A simple method to estimate relative stabilities of polyethers cationized by alkali metal ions. Rapid Commun. Mass Spectrom. 26, 304-308 (2012)

38. Memboeuf, A., Nasioudis, A., Indelicato, S., Pollreisz, F., Kuki, Á., Kéki, S., Van Den Brink, O.F., Vékey, K., Drahos, L.: Size effect on fragmentation in tandem mass spectrometry. Anal. Chem. 82, 2294-2302 (2010)

39. Baumgaertel, A., Altuntaș, E., Kempe, K., Crecelius, A., Schubert, U.S.: Characterization of different poly(2-oxazoline) block copolymers by MALDI-TOF MS/MS and ESI-Q-TOF MS/MS. J. Polym. Sci. Part A Polym. Chem. 48, 5533-5540 (2010)

40. Kuki, Á., Nagy, L., Memboeuf, A., Drahos, L., Vékey, K., Zsuga, M. Kéki, S.: Energy-dependent collision-induced dissociation of lithiated polytetrahydrofuran: effect of the size on the fragmentation properties. J. Am. Soc. Mass Spectrom. 21, 1753-1761 (2010)
41. Haler, J.R.N., Massonnet, P., Far, J., de la Rosa, V.R., Lecomte, P., Hoogenboom, R., Jérôme, C., De Pauw, E.: Gas-phase dynamics of collision induced unfolding, collision induced dissociation, and electron transfer dissociation-activated polymer ions. J. Am. Soc. Mass Spectrom. $1-10$ (2018)

42. Larriba, C., Fernandez De La Mora, J.: The gas phase structure of coulombically stretched polyethylene glycol ions. J. Phys. Chem. B. 116, 593-598 (2012)

43. De Winter, J., Lemaur, V., Ballivian, R., Chirot, F., Coulembier, O., Antoine, R., Lemoine, J., Cornil, J., Dubois, P., Dugourd, P., Gerbaux, P.: Size dependence of the folding of multiply charged sodium cationized polylactides revealed by ion mobility mass spectrometry and molecular modelling. Chem. - A Eur. J. 17, 9738-9745 (2011)

44. Haler, J.R.N., Morsa, D., Lecomte, P., Jérôme, C., Far, J., De Pauw, E.: Predicting ion mobility-mass spectrometry trends of polymers using the concept of apparent densities. Methods. 144, 125-133 (2018)

45. Trimpin, S., Plasencia, M., Isailovic, D., Clemmer, D.E.: Resolving oligomers from fully grown polymers with IMS-MS. Anal. Chem. 79, 7965-7974 (2007)

46. Kune, C., Far, J., De Pauw, E.: Accurate drift time determination by traveling wave ion mobility spectrometry: the concept of the diffusion calibration. Anal. Chem. 88, 11639-11646 (2016)

47. Goossens, H., Catak, S., Glassner, M., de la Rosa, V.R., Monnery, B.D., De Proft, F., Van Speybroeck, V., Hoogenboom, R.: Cationic ringopening polymerization of 2-propyl-2-oxazolines: understanding structural effects on polymerization behavior based on molecular modeling. ACS Macro Lett. 2, 651-654 (2013)

48. Shemirani, G., Kéki, S.: Collision Induced Dissociation Study of Polyethers, (2015) 